\title{
Imminent ischemia in normal and hypertrophic Langendorff rat hearts; effects of fatty acids and superoxyde dismutase monitored by NADH surface fluorescence
}

\author{
W.C. Hulsmann ${ }^{b}$, J.F. Ashruf ${ }^{a}$, H.A. Bruining ${ }^{a}$ and C. Ince ${ }^{a}$ \\ a Department of surgery and ${ }^{b}$ Thorax centre, Erasmus University, Rotterdam (The Netherlands) \\ (Received 13 August 1992) \\ (Revised manuscript received 25 January 1993)
}

Key words: Ischemia; NADH-fluorescence; Fatty acid; Oxygen radical; (Rat heart)

\begin{abstract}
Hypertrophic hearts contain areas of hypoperfusion which can be visualized by increased NADH surface fluorescence during in vitro perfusion without oxygen-carrying particles under constant pressure and pacing. By contrast, fluorescence remained low when non-hypertrophic hearts were used instead. When during perfusion of normal hearts the pH of the medium was lowered from 7.5 to 7.0, areas of high fluorescence appeared in a few minutes. The high fluorescent areas under conditions of cardiac hypertrophy or $\mathrm{pH} 7.0$ perfusion could be reduced by addition of superoxide dismutase. It indicates that oxygen free radicals interfere with proper flow regulation in areas of low pH. Fluorescence in hypertrophic hearts also diminished during addition of albumin-bound oleate to the standard, glucose-containing, medium. This is in agreement with our earlier finding of fatty acid protection from acidosis-initiated loss of capillary flow (Biochim. Biophys. Acta, 1033 (1990) 214-218). In contrast to low concentrations of free fatty acids, high concentrations interfere with tissue oxygenation. This has been illustrated by the use of 1 $\mathrm{mM}$ octanoate, which after a few min caused the appearance of high fluorescent areas. We conclude that decompensation of flow in hypoperfused areas of heart, as occurs in hypertrophy, may be stimulated by acidosis and oxygen free radicals.
\end{abstract}

\section{Introduction}

Previous work has shown that the Langendorff rat heart, when paced at a rate of 300 beats/min, but perfused with neither erythrocytes nor with fluorocarbon as oxygen carriers, is borderline aerobic, as concluded from the continuous release of lactate. Glyco(geno)lysis is inhibited by increase of $\mathrm{O}_{2}$ supply by fluorocarbon addition $[1,2]$. Lowering of the $\mathrm{pH}$ of the perfusion medium results in loss of high affinity binding of $\mathrm{Ca}^{2+}$ to membranes [3-5]. This results in a brief period of positive inotropy, accompanied by an increase of flow rate, followed by progressive decline of inotropy and flow so that ischemia follows $[1,6,7]$. This phenomenon may be delayed by fatty acids $[6,7]$, which are known to have vasodilatory properties (compare ref. 8). Development of cardiac ischemia may be studied by NADH fluorescence [6,9-11]. It allows the detection of small areas in which the ischemia begins so that early intervention of imminent ischemia may be

Correspondence to: C. Ince, Department of Surgery, Erasmus University Rotterdam, Dr. Molewaterplein 40, 3015 GD Rotterdam, The Netherlands. studied. We have selected to study the effect of superoxide dismutase (SOD) as we found earlier that cardiac function, lost by hypoperfusion of Langendorff hearts, improves by SOD addition by the removal of oxygen free radicals (OFR) from the interstitium [12]. In the present investigation we also studied hypoperfusion in normal hearts by lowering the perfusion $\mathrm{pH}$, in order to induce interstitial acidosis which may be involved in the progression of cardiac hypertrophy. These hearts contain hypoperfused areas [13] in which local acidosis, due to enhanced glycolysis, may be present. As the heterogeneous distribution of (imminent) ischemia make data of overall metabolic changes difficult to interpret, we refrained from determining metabolic parameters in the present investigation.

\section{Materials and Methods}

Male Wistar rats weighing between 220 and $320 \mathrm{~g}$ were used. The hearts were removed, cooled in cold perfusion medium and rapidly arranged for perfusion at $37^{\circ} \mathrm{C}$ and a pressure of $8.3 \mathrm{kPa}$ according to the Langendorff technique with a medium consisting of $128 \mathrm{mM} \mathrm{NaCl}, 4.7 \mathrm{mM} \mathrm{KCl}, 1 \mathrm{mM} \mathrm{MgCl} 2,0.4 \mathrm{mM}$

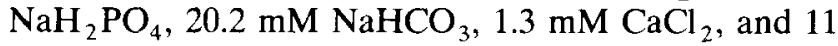


$\mathrm{mM}$ glucose [8]. Other additions are described under Results. All hearts were paced at $5 \mathrm{~Hz}$. NADH surface fluorescence was done after excitation of about $1 \mathrm{~cm}^{2}$ of the left ventricle of Langendorff rat hearts with light of $360 \mathrm{~nm}$ and the emitted light $(460-490 \mathrm{~nm})$ registered as described [10]. Images were recorded with a S-VHS Panasonic video-recorder and computer-analyzed [10]. Normal hearts had a total ventricle weight of $1.00 \pm 0.04 \mathrm{~g}(n=10)$ and hypertrophic hearts (obtained after suprarenal abdominal aorta banding; 15) of $1.52 \pm 0.06 \mathrm{~g}(n=10)$. The values are mean values \pm standard error of the mean (S.E.M.). Oleate-containing medium was used when indicated and prepared by sonicating oleic acid with an equivalent amount of $\mathrm{NaOH}$. The warm soap was added to fatty acid-free bovine serum albumin (molar ratio fatty acid to albumin $4: 1$ ). The freshly prepared stock solution was added to the other components of the perfusion buffer and filtered through Millipore filter $(0.45 \mu \mathrm{m})$ and 0.1 mM L-carnitine (a gift from Sigma Tau, Pomezia, Italy) was added. Most chemicals were from E. Merck (Darmstadt, Germany); SOD was from Boehringer (Mannheim, Germany).

\section{Results}

\section{'Reducing power' of various substrates in Langendorff hearts}

During perfusion of the paced rat Langendorff heart with standard perfusion medium $(\mathrm{pH} 7.5)$, containing $11 \mathrm{mM}$ glucose as substrate, NADH fluorescence of a representative area of the left ventricle was measured, and the acute change by adding various substrates was registered. Fig. 1 shows that the increase of NADH fluorescence by additional substrates tested (1-2 mM) decreased in the order: pyruvate $>$ oleate $>$ octanoate $>$ lactate $>$ beta-hydroxybutyrate/acetoacetate. Average values of $n=3$ are presented. This led us to select pyruvate, octanoate and oleate in the present studies, as we assumed that an optimal supply of reducing equivalents to cardiomyocytes could limit breakdown of ATP and generation of (hypo)xanthine, substrate for (endothelial) xanthine oxidase-dependent $\mathrm{O}_{2}$-generation. That the addition of pyruvate increases NADH fluorescence confirms that the NADH fluorescence observed is of mitochondrial and not of cytosolic origin [9], as in the latter case it would have declined instead, due to stimulation of lactate dehydrogenase activity.

Sufficient $\mathrm{O}_{2}$ supply disrupted by acidosis; effects of respiratory substrates and superoxyde dismutase (SOD)

As mentioned earlier, changing the $\mathrm{pH}$ of the standard perfusion medium from 7.5 to 7.0 rapidly results in a decrease of coronary flow and contractility of the heart (after an initial increase of both parameters (compare ref. 7)). This is accompanied by the appear-

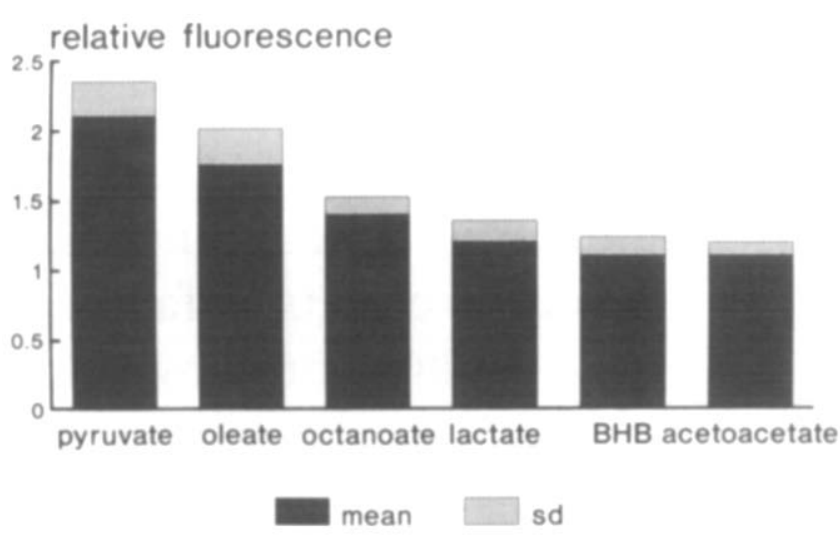

Fig. 1. Comparison of the NADH fluorescence of normal Langendorff hearts perfused with the substrates indicated (in addition to glucose), with the NADH fluorescence when perfused with glucose as the sole substrate $(n=3$ : mean \pm S.D. $)$.

ance of areas of high fluorescence (Fig. 2A,B). Addition of pyruvate was not able to prevent $\mathrm{pH} 7.0$ induced ischemia (not shown; $n=4$ ). When SOD had been present in the $\mathrm{pH} 7.5$ medium, the change to the pH 7.0 medium still led to immediate and strong increase of NADH fluorescence in large parts of the heart, which persisted. However, it gradually decreased when the SOD supply was also contained in the $\mathrm{pH} 7.0$ medium (Fig. 3). When after $30 \mathrm{~min}$ SOD in pH 7.0 medium, $\mathrm{pH} 7.0$ perfusion without SOD was continued, no fluorescence increase was observed after 15 min, suggestive for continued SOD activity in the interstitium when SOD supply was discontinued (not shown). The flow, that had decreased from about 8 $\mathrm{ml} / \mathrm{min}$ per $\mathrm{g}$ (after $20 \mathrm{~min}$ perfusion in $\mathrm{pH} 7.5$ medium) to $1.9 \pm 0.7 \mathrm{ml} / \mathrm{min}$ per $\mathrm{g}(n=6)$ after 15 min $\mathrm{pH} 7.0$ perfusion without SOD, generally did not
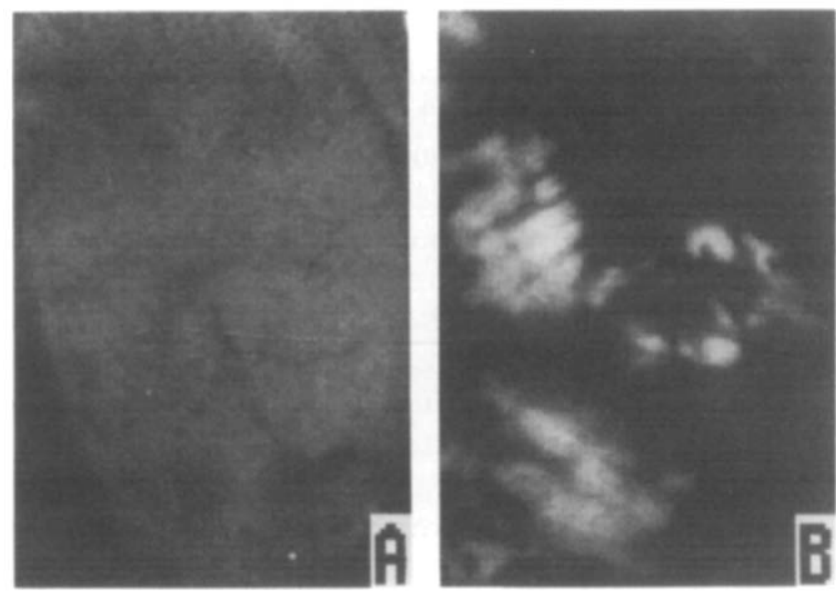

Fig. 2. Effect of pH 7.0 perfusion on the NADH fluorescence of a normal heart: (A) a heart during $\mathrm{pH} 7.5$ perfusion; no ischemia is present as witnessed by the low fluorescence of the displayed left ventricle surface and (B) the same heart 3 min after switching from pH 7.5 to $\mathrm{pH} 7.0$ perfusion; the white areas represent high NADH fluorescent ischemic areas. 

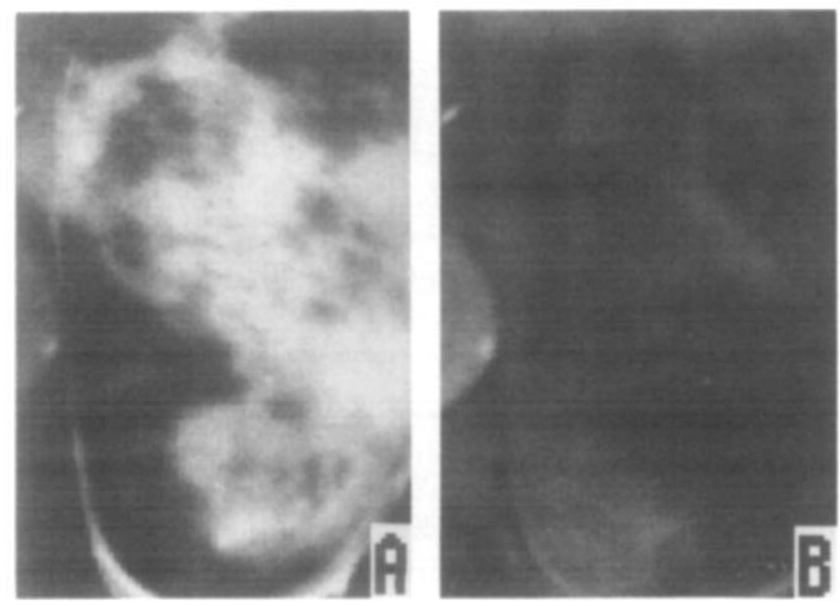

Fig. 3. Effect of SOD addition to the medium on ischemic areas induced by $\mathrm{pH} 7.0$ perfusion: (A) a heart after $5 \mathrm{~min} \mathrm{pH} 7.0$ perfusion and (B) the same heart $15 \mathrm{~min}$ after $\mathrm{pH} 7.0$ perfusion with $10 \mathrm{mg} / \mathrm{I} \mathrm{SOD}$ in the perfusate.

drop when SOD was present (flow: $8.6 \pm 2.5 \mathrm{ml} / \mathrm{min}$ per $\mathrm{g} ; n=4)$. The large variation may be due to the overall heterogeneity of the phenomena. We interpreted the absence of SOD effect when it was only supplied during $\mathrm{pH} 7.5$ perfusion by its inability to penetrate into the interstitium when interendothelial junctions are tight. During $\mathrm{pH} 7.0$ perfusion, SOD entry into the interstitium must have occurred, perhaps due to increased permeability by $\mathrm{pH}$ lowering, as will be discussed.

\section{Octanoate addition and NADH fluorescence}

Our previous experience [7], that albumin-bound oleate can prevent the strong decrease of flow by changing the $\mathrm{pH}$ of the perfusion medium from 7.5 to 7.0 , led us to use another fatty acid, octanoate, in the present study. This fatty acid as well as acetate has been shown by others to protect the energy state of glucose-perfused working rat hearts after respiratory acidosis [6]. Under our conditions, changing of the standard perfusion medium of $\mathrm{pH} 7.5$ (with glucose) to one containing in addition $1 \mathrm{mM}$ octanoate caused an immediate overall increase of fluorescence (cf. Fig. 1). After a few min, areas of high NADH fluorescence became visible, which gradually increased in size (Fig. 4). This phenomenon appeared to be reversible by continued perfusion without octanoate, requiring at least $30 \mathrm{~min}$. This experiment was repeated thrice with similar result. Therefore $1 \mathrm{mM}$ final concentration of octanoate probably resulted in redistribution of flow, which we had never seen when using oleate or pyruvate at $\mathrm{pH} 7.5$ instead. In the latter cases a moderate, but evenly distributed fluorescence increase was seen, which served to construct Fig. 1. When during octanoate addition the $\mathrm{pH}$ of the medium was switched from 7.5 to 7.0 , the flow rapidly deteriorated, which resulted in increase of NADH fluorescence (Fig. 5).

\section{Hypertrophic hearts and NADH fluorescence}

From the start of Langendorff perfusion ( $\mathrm{pH} 7.5$ and glucose as the only substrate), hypertrophic hearts show areas of high NADH fluorescence, that gradually increase in size and intensity. Fig. 6 shows that hypertrophy is not a homogeneous phenomenon, probably due to different sarcomere/capillary ratios in different areas [13]. The increase of fluorescence can be stopped and acutely reversed by the addition of vasodilators, like nitroprusside or adenosine, or by improvement of tissue oxygenation by fluorocarbon [11]. Addition of 0.8 $\mathrm{mM}$ oleate (containing $0.2 \mathrm{mM}$ bovine serum albumin and $0.1 \mathrm{mM}$ carnitine) resulted in a gradual decrease of the fluorescent areas (Fig. 7; two examples of four experiments with similar effect). SOD (final concentration $10 \mathrm{mg} / \mathrm{l}$ ) added instead of oleate/albumin/carni-
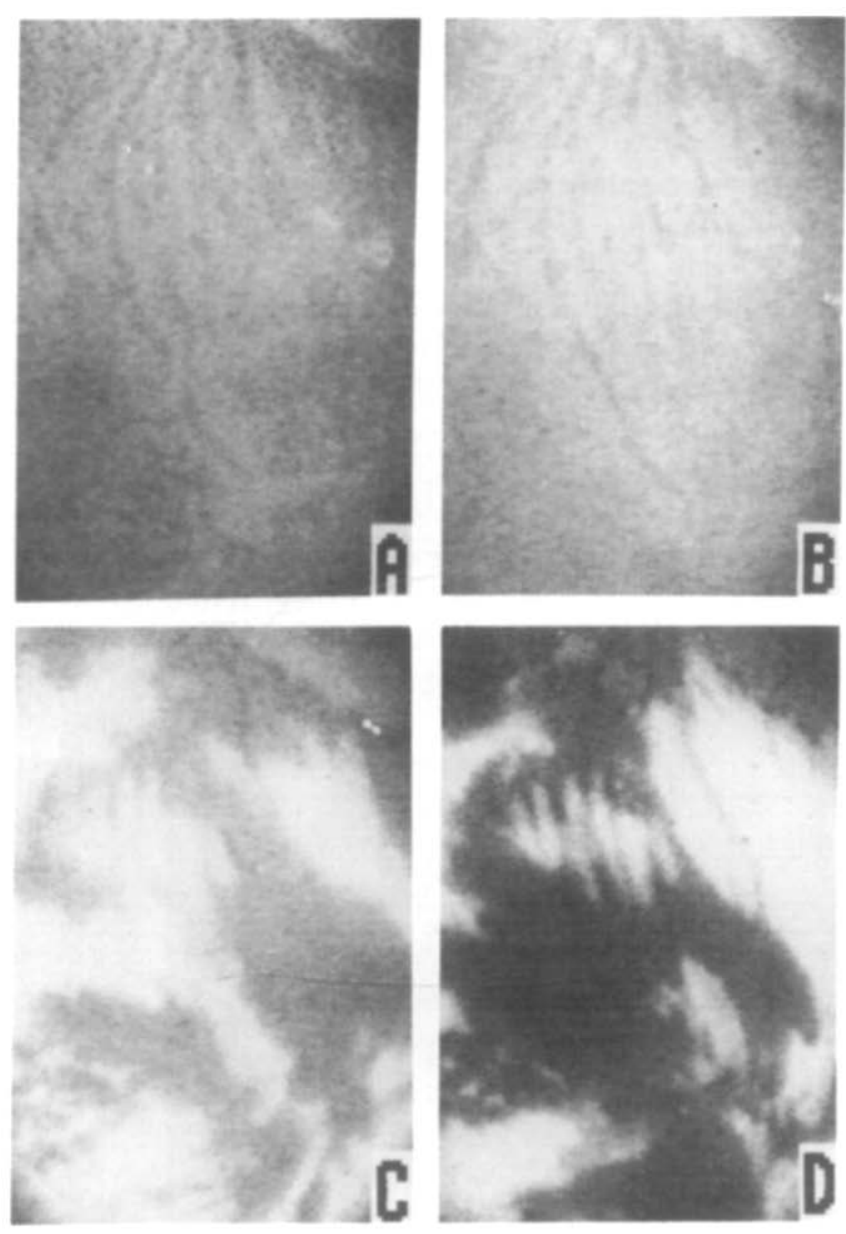

Fig. 4. Effect of octanoate addition to the medium on NADH fluorescence of a normal heart: (A) prior to octanoate addition, (B) 1 min after octanoate addition; there is a overall increase of NADH fluorescence, (C) $10 \mathrm{~min}$ after octanoate addition; large ischemic areas are present and (D) $10 \mathrm{~min}$ after switching back to perfusion without octanoate. 


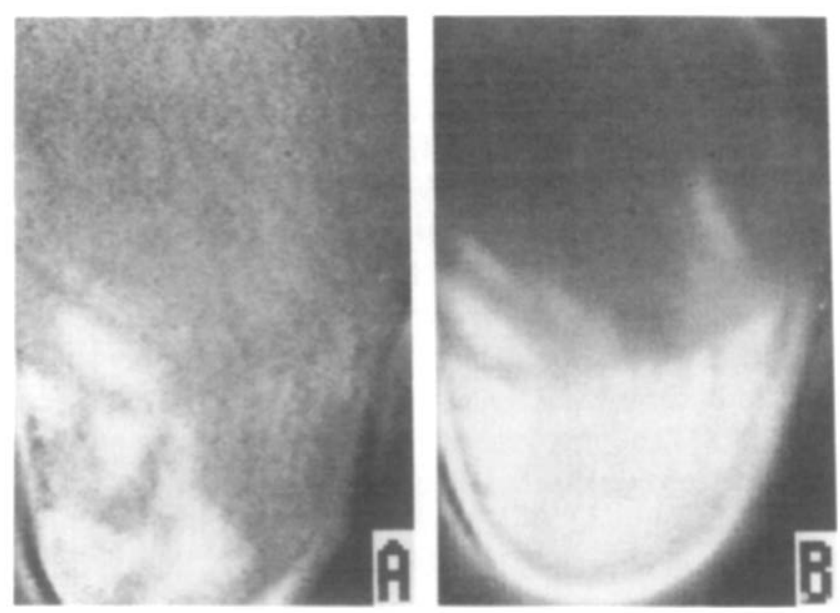

Fig. 5. Effect of $\mathrm{pH}$ change upon a normal heart perfused with octanoate containing medium: (A) a heart during $\mathrm{pH} 7.5$ perfusion with octanoate; note the ischemic areas and (B) the same heart 1 min after switching to $\mathrm{pH} 7.0$ perfusion; the ischemic area has rapidly increased in size.

tine also gradually diminished NADH fluorescence. Whereas the disappearance of ischemia by nitroprusside, adenosine or fluorocarbon was a matter of seconds, the effects of oleate/albumin/carnitine or SOD was a matter of minutes. The effect of SOD required a lag phase of about $15 \mathrm{~min}$. Fig. 8 shows two examples out of four with similar effect (in a fifth experiment no effect was observed after $30 \mathrm{~min}$ SOD perfusion). When SOD addition was stopped after $30 \mathrm{~min}$, fluorescence remained low $(n=4)$ for at least $30 \mathrm{~min}$, suggesting persistent SOD activity in the interstitium.

\section{Discussion}

The present work indicates that in the absence of oxygen-carrying particles, the $\mathrm{O}_{2}$-saturated perfusion medium only provides borderline aerobiosis in paced normal Langendorff hearts. This is reflected by a low production rate of lactate [1,2], while NADH fluorescence is low. Fluorescence rapidly increases when the $\mathrm{pH}$ of the medium is lowered to 7.0 or less, indicative of decreased flow in affected areas, as may have been expected from previous investigations in which either respiratory acidosis was effected in working hearts $[6,14]$ or metabolic acidosis in Langendorff hearts [1.7]. The heterogeneous pattern of fluorescence increase by acidosis, induced by changing the $\mathrm{pH}$ of the medium from 7.5 to 7.0 during Langendorff perfusion in the present work (Fig. 2), could be due to differences in the regulation of flow in different areas of the heart. Also, in hypertrophic hearts perfused with standard medium ( $\mathrm{pH} 7.5$ ) the appearance of heterogeneously distributed high fluorescent areas became visible [11]. They rapidly disappear when the coronary flow is increased by the addition of nitroprusside or adenosine [11]. Apparently, in those areas flow decreases progressively in the absence of added vasodilators so that a critical $\mathrm{O}_{2}$ level is quickly reached in which NADH of the mitochondrial respiratory chain accumulates. This is in agreement with the disappearance of $\mathrm{NADH}$ fluorescence by increasing the $\mathrm{O}_{2}$ supply by addition of fluorocarbon to the perfusion medium [11]. This also limits lactic acid formation during $\mathrm{pH} 7.0$ perfusion [1]. These observations suggest that local acidosis is a common factor involved in the decline of flow in certain areas of $\mathrm{pH}$ 7.0-perfused control hearts or $\mathrm{pH} 7.5$ perfused hypertrophic hearts. Steenbergen et al. [14] observed that "acidosis-induced ischemia is characterized by relatively large areas of high fluorescence at opposed to the mottled appearance of high flow hypoxia". They concluded from this that changes of coronary circulation are important for the development of the anoxic zones [14]. Interestingly, mild acidosis may result in vasodilation, but severe acidosis constricts
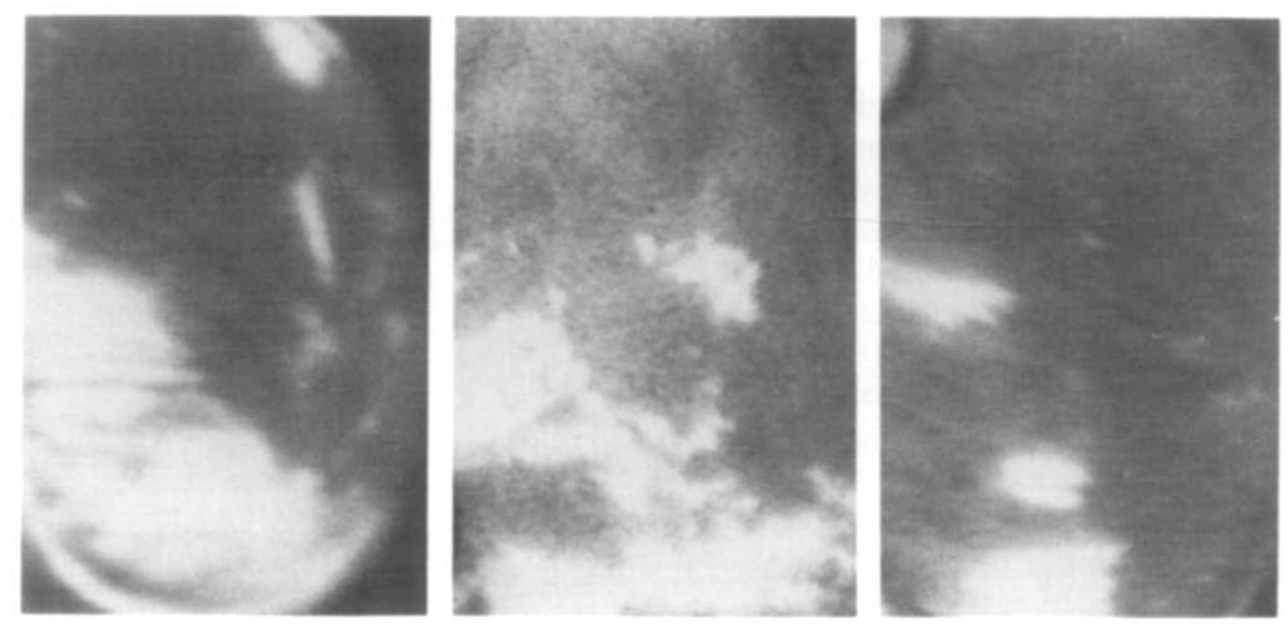

Fig. 6. Three examples of hypertrophic Langendorff hearts. The white areas on the left ventricle surface are ischemic areas which developed within $5 \mathrm{~min}$ after the start of perfusion. 
vessels. As for acidosis and vasodilation, we observed that initially, after changing $\mathrm{pH} 7.5$ to $\mathrm{pH} 7.0$ perfusion, the contractility of non-working Langendorff hearts increased together with coronary flow [7]. Only after contractility had started to fall, did the increased flow gradually diminish and finally resulted in low flow ischemia, probably due to further decline of the $\mathrm{pH}$ in the tissue by lactic acid formation and ATP breakdown $(1,7)$. Fatty acids also have dual effects on flow, depending on their free concentration. Low levels of long-chain fatty acids, bound to albumin, are vasodilators [8]. The initial effect of $1 \mathrm{mM}$ octanoate on overall flow during $\mathrm{pH} 7.5$ perfusion was also an increase $(45 \pm 8 \%, n=4$, present study; compare also ref. 8$)$ ). However, after $5 \mathrm{~min}$ the overall flow had started to fall, while ischemic areas were formed (Fig. 4). When the octanoate-containing medium of $\mathrm{pH} 7.5$ was changed into one of $\mathrm{pH} \mathrm{7.0,} \mathrm{the} \mathrm{ischemic} \mathrm{area} \mathrm{largely}$ increased within 5 min (Fig. 5), while the overall flow dropped to low values $(1-3 \mathrm{ml} / \mathrm{min}$ per $\mathrm{g})$. It suggests that the degree of fatty acid dissociation plays a role in their effectiveness to penetrate membranes. It has been
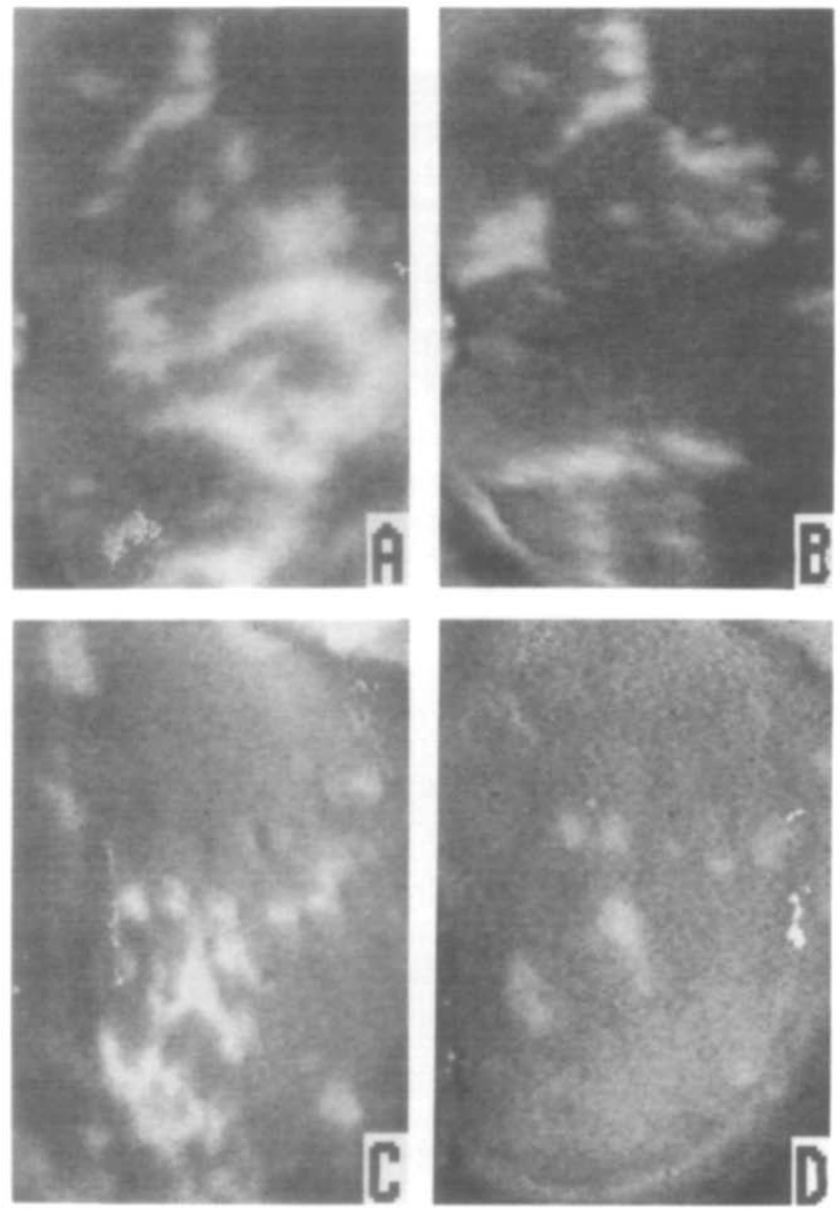

Fig. 7. Effect of oleate addition to the perfusion medium on ischemic areas of hypertrophic hearts: (A) and (B) are images of 2 different hearts prior to oleate addition; (C) and (D) display the same hearts as (A) and (B) respectively, $2 \mathrm{~min}$ after switching to perfusion containing oleate.
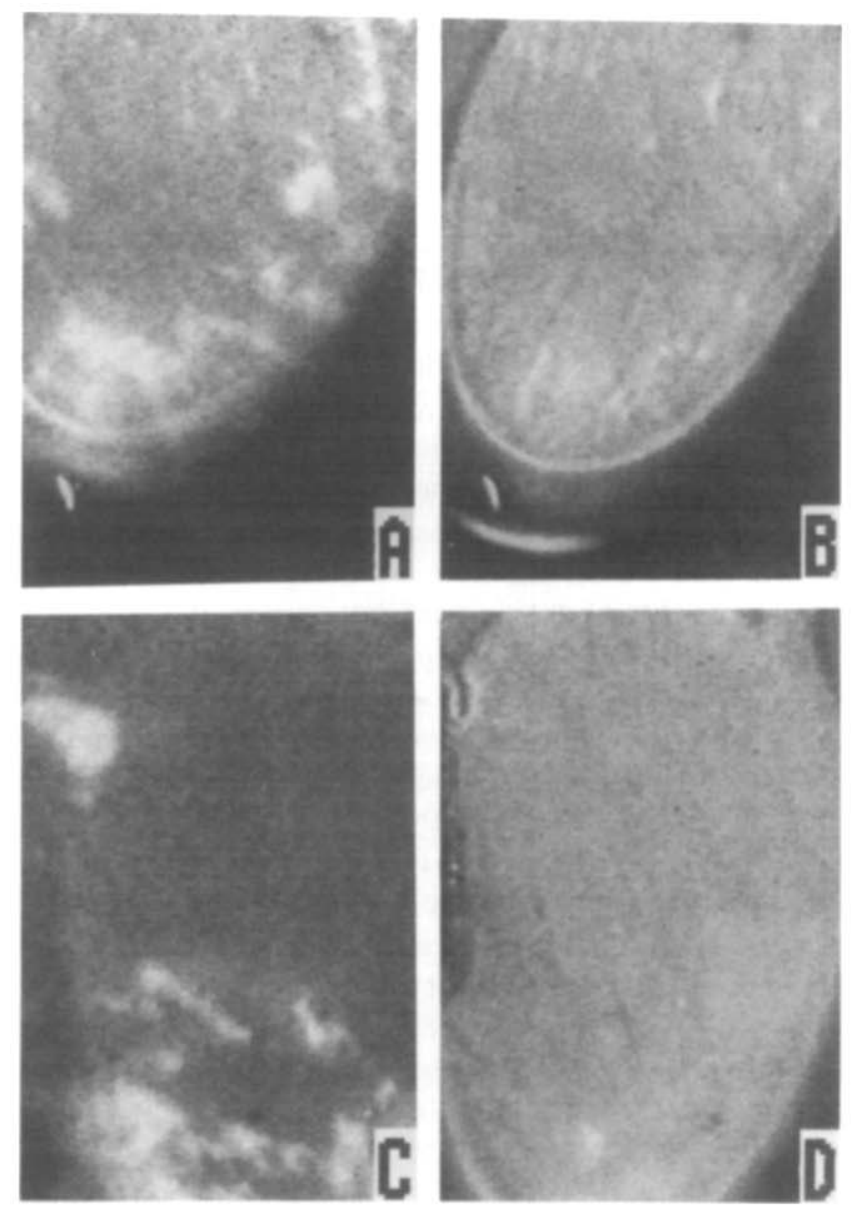

Fig. 8. Effect of SOD addition to the perfusion medium on ischemic areas of hypertrophic hearts: (A) and (B) are images of 2 different hearts prior to SOD addition; (C) and (D) display the same hearts as (A) and (B) respectively, $15 \mathrm{~min}$ after perfusion with SOD (10 mg/l) containing medium.

shown that the effect of amphiphiles depends on the charge of these membrane-active agents [16]. Fatty acids and lysophospholipids have (indirect) ionophoric properties (compare refs. 8 and 16-18) which can affect the $\mathrm{Ca}^{2+}$ level in cells. In smooth muscle- and endothelial cells of blood vessels, amphiphiles affect the contractile state of these cells and influence capillary flow. They may also cause edema, as had been seen earlier by employing $5 \mathrm{mM}$ octanoate during $\mathrm{pH}$ 7.5 Langendorff perfusion. After an initial flow increase, the flow rate progressively decreased, which was accompanied by the release of lactate, adenosine, inosine and hypoxanthine, suggestive of insufficient oxygenation [8]. In those experiments macroscopic edema appeared, judged by the shiny appearance of the heart and increase of its size. In the present experiments in which $1 \mathrm{mM}$ octanoate was used instead, macroscopic edema did not occur during $30 \mathrm{~min}$ perfusion. Yet, extravasation of fluid may have been present as restoration of low fluorescence by octanoate-free perfusion only slowly occurred during a 30-min further observation. 
The interstitial space plays an important role in the initiation of cardiac hypertrophy [19]. Increase of the permeability of endothelium by fatty acids and/or acidosis has been described by many authors. A relation between protons and fatty acids on permeability of capillaries may be the natural presence of fatty acids in membranes, which at $\mathrm{pH} 7.4$ are mainly in ionized form and as such part of the bilayer structure. However, upon acidification, unionized species may escape from lamellar structures $[20,21]$ and activate membrane processes, that result in increased intracellular $\mathrm{Ca}^{2+}$ levels. The increased penetration of vascular constituents into the interstitial space may also be the reason why SOD was able to enter the interstitium during $\mathrm{pH} 7.0$ perfusion and not during the previous pH 7.5 perfusion in the experiments shown in Fig. 3 . These illustrate improvement of flow during acidosis by SOD. The benificial effect of SOD on tissue oxygenation also applies to $\mathrm{pH} 7.5$ perfused hypertrophic hearts (Fig. 8). It indicates also that in hypertrophic hearts extracellular OFR interferes with the regulation of capillary flow. This could be due to the ability of OFR to destroy endothelium-derived relaxing factor [22]. A low rate of secretion and/or adequate removal of OFR from the outside of endothelial cells is most important to prevent ischemia. This may also be the basis of the inhibitory effect of albumin-complexed oleate upon the progression of flow decrease during $\mathrm{pH} 7.0$ perfusion of normal hearts [7] and to $\mathrm{pH}$ 7.5-perfused hypertrophic hearts, in which hypoperfusion may even disappear under the conditions of the tests (Fig. 7). The explanation may be that during fatty acid oxidation in the (peri)vascular area, in which $\mathrm{O}_{2}$ is still present, lactic acid formation is inhibited and endothelium-derived relaxing factor is increased [23].

\section{Acknowledgements}

Mr. R. Cornelissen, Department of Physiology, University of Limburg, The Netherlands, is thanked for preparing the aortic banding of rats for generation of cardiac hypertrophy.

\section{References}

I Hulsmann, W.C. and De Wit, L.E.A. (1990) (ell Biol. Int. Rep. $14,311-315$.

2 Hulsmann, W.C., De Wit, L.E.A., Stam, H. and Schoonderwoerd, K. (1990) Biochim. Biophys. Acta 1055, 189-192.

3 Williamson, J.R., Safer, B., Rich, T., Schaffer, W. and Kobayashi. K. (1975) Acta Med. Scand. Suppl. 587, 95-111.

4 Vandeplassche, G. and Borgers, M. (1990) Cell. Biol. Int. Rep. $14,317-334$

5 Kwan, C.Y. and Daniel, E.E. (1989) in Calcium in essential hypertension (Aoli, K. and E.D. Frohlich, E.D., eds.), pp. $201-$ 230, Academic Press Japan.

6 Schaffer, S.W., Safer, B., Ford, C., Illingworth, J. and Williamson. J.R. (1978) Am. J. Physiol. 234, H40-H5I.

7 Hulsmann, W.C., De Wit, L.E.A., Schneydenberg, C. and Verkleij, A.J. (1990) Biochim. Biophys. Acta 1033, 214-218.

8 Hulsmann, W.C. (1976) Basic Res. Cardiol. 71. 179-191

9 Chance, B. (1976) Circ. Res. 38 suppl. I, 31-38.

10 Ince, C.. Ashruf, J.F., Avontuur, J.A.M., Wieringa, P.A., Spaan. J.A.E. and Bruining, H.A. (1993) Am. J. Physiol. 264, H294-H301.

11 Ashruf, J.F., Ince, C.. Bruining, H.A. and Hulsmann, W.C. (1993) Adv. Exp. Med. Biol., in press.

12 Hulsmann, W.C. and Dubelaar, M.L. (1987) Cardiovasc. Res. 21. 674-677.

13 Katz, A.M. (1990) N. Engl. J. Med. 323, 100-110.

14 Steenbergen, C., Deleeuw, G., Barlow, C.. Chance, B. and Williamson, J.R. (1977) Circ. Res. 606-615.

15 Malik, A.B., Shapiro, J.M., Yanics, J., Rojales, A. and Geha, A.S. (1974) Cardiovasc. Res. 8, 801-805.

16 Epand, R.M. and Lester, D.S. (1990) TIPS 11, 317-321.

17 Kirber, M.T., Ordway, R.W., Clapp, L.H., Walsh, J.V. and Singer. J.J. (1992), FEBS Lett. 297, 24-28.

18 Locher, R., Weisser, B., Mengden, I., Brunner, C. and Vetter, W. (1992) Biochim. Biophys. Res. Commun. 183, 156-162.

19 Allen, G.A. and Laine, S.J. (1991) Circ. Res. 68, 1713-1721.

20 Amende, L.M., Blanchette-Mackie, J.J., Chernick, and Scow. R.O. (1985) Biochim. Biophys. Acta 837, 94-102.

21 Cistola, D.P., Hamilton, J.A., Jackson, D., Small, D.M. (1988) Biochemistry 27, 1881-1888.

22 Moncada, S., Palmer, R.M.J. and Higgs, E.A. (1991) Pharmacol Rev. 43, 109-142.

23 Hulsmann, W.C. and Dubelaar, M.L. (1992) Mol. Cell. Biochemistry $116,125-129$ 